\title{
Expanded Multi-Band Super-Nyquist CAP Modulation for Highly Bandlimited Organic Visible Light Communications
}

\author{
Petr Chvojka, Paul Anthony Haigh, Alessandro Minotto, Andrew Burton, Petri Murto, Zewdneh Genene, \\ Wendimagegn Mammo, Mats R. Andersson, Ergang Wang, Zabih Ghassemlooy, Franco Cacialli, Izzat Darwazeh \\ and Stanislav Zvanovec
}

\begin{abstract}
In this paper, we experimentally demonstrate a novel expanded non-orthogonal multi-band super-Nyquist carrier-less amplitude and phase ( $m$-ESCAP) modulation for bandlimited organic visible light communication (VLC) systems. The proposed
\end{abstract}

Manuscript received $\mathrm{mm} / \mathrm{dd} / \mathrm{yyyy}$.

P. C. and P. A. H. contributed equally to this work. This work was supported in part by the UK EPSRC grant, EP/P006280/1: Multifunctional Polymer Light-Emitting Diodes with Visible Light Communications (MARVEL) and in part by the European Union's Horizon 2020 research and innovation programme under the Marie Sklodowska-Curie grant agreement no 764461 (VISION). E. W. acknowledges the Swedish Research Council and the Swedish Research Council Formas, the Wallenberg Foundation through the Wallenberg Academy Fellows program for financial support. W. M. and Z. G. gratefully acknowledge financial support from the International Science Program (ISP), Uppsala University, Sweden. F. C. is a recipient of a Royal Society Wolfson Foundation Research Merit Award.

P. Chvojka and S. Zvanovec are with the Department of Electromagnetic Field, Faculty of Electrical Engineering, Czech Technical University in Prague, Technicka 2, 16627, Prague, Czech Republic (e-mail: petr.chvojka@fel.cvut.cz; xzvanove@fel.cvut.cz).

I. Darwazeh is with the Communications \& Information Systems Group, Department of Electrical \& Electronic Engineering, University College London, London, WC1E 6BT, UK (e-mail: i.darwazeh@ucl.ac.uk).

P. A. Haigh is with the School of Engineering, Newcastle University, Newcastle upon Tyne, NE1 7RU, UK. P.A.H. was with the Communications \& Information Systems Group, Department of Electrical \& Electronic Engineering, University College London, London, WC1E 6BT, UK and with the Photonics Innovations Laboratory (Pi-Lab), Department of Electrical \& Electronic Engineering, University College London, London WC1E 6BT, UK (e-mail: p.haigh@ucl.ac.uk).

A. Minotto and F. Cacialli are with the Department of Physics and Astronomy and the London Centre for Nanotechnology, University College London, Gower Street, London WC1E 6BT, UK (e-mail: a.minotto@ucl.ac.uk; f.cacialli@ucl.ac.uk).

P. Murto is with the Department of Chemistry and Chemical Engineering/Applied Chemistry, Chalmers University of Technology, Gothenburg SE412 96, Sweden and with the Flinders Institute for NanoScale Science \& Technology, Flinders University, Adelaide, SA 5042, Australia (e-mail: murto@chalmers.se).

Z. Genene is with the Department of Chemistry, Addis Ababa University, Addis Ababa, P.O. Box 33658, Ethiopia and with the Department of Chemistry, Ambo University, P.O. Box 19, Ambo, Ethiopia (e-mail: zewdneh.genene@ambou.edu.et).

W. Mammo is with the Department of Chemistry, Addis Ababa University, Addis Ababa, P.O. Box 33658, Ethiopia (e-mail: wendimagegn.mammo@aau.edu.et).

M. R. Andersson is with the Flinders Institute for NanoScale Science \& Technology, Flinders University, Adelaide, SA 5042, Australia (e-mail: mats.andersson@ flinders.edu.au).

E. Wang is with the Department of Chemistry and Chemical Engineering/Applied Chemistry, Chalmers University of Technology, Gothenburg SE412 96, Sweden (e-mail: ergang@ chalmers.se).

Z. Ghassemlooy and A. Burton are with the Optical Communications Research Group, Faculty of Engineering and Environment, Northumbria University, Newcastle upon Tyne, NE1 8ST, UK (e-mail: z.ghassemlooy@northumbria.ac.uk; andrew2.burton@northumbria.ac.uk). scheme has the same bandwidth requirement as the conventional $m$-CAP while breaking the orthogonality between subcarriers by purposely overlapping them. We compare $m$-ESCAP with the conventional $m$-CAP and a compressed non-orthogonal version of $m$-CAP ( $m$-SCAP) in terms of measured bit error rate (BER) performance, bit rates and spectral efficiencies. We show that the $m$-ESCAP system offers improvement in the bit rate of $\sim 10 \%$ and $20 \%$ compared to the $m$-CAP and $m$-SCAP, respectively, and in the spectral efficiency of $\sim 20 \%$ compared to $m$-CAP. These gains are achieved at the cost of increased BER, which however remains below the $7 \%$ forward error correction limit.

Index Terms - digital signal processing, multi-band carrier-less amplitude and phase modulation, non-orthogonal, organic light emitting diode, visible light communications.

\section{INTRODUCTION}

$\mathbf{T}$ HE effective utilization of the transmission bandwidth is a challenging task in designing future robust high data rate communication systems. Besides the widely used orthogonal frequency division multiplexing (OFDM) scheme, carrierless amplitude and phase (CAP) modulation and its multi-band variant (i.e., $m$-CAP) have both been adopted in visible light communication (VLC) systems recently to support both higher transmission speed and spectral efficiency [1]-[5]. In addition, a number of signal processing techniques have been developed to further improve a VLC system performance, which is limited predominantly by the inherent modulation bandwidth of the white light-emitting diodes (LEDs) used as transmitters. Namely, in spectrally efficient FDM (SEFDM) the individual subcarriers are squeezed closer together in comparison to OFDM, thus breaching the orthogonality requirement in order to save bandwidth in both optical and electrical domains at the cost of increased receiver complexity due to inter-carrier interference (ICI) [6], [7]. Contrary to SEFDM, fast OFDM (FOFDM) maintains the orthogonality between subcarriers with a spacing of $1 / 2 T$, where $T$ is the symbol interval, while occupying a half of the bandwidth of OFDM, but restricting the use of symbol alphabets to real-values [8], [9]. In [10], [11], faster-than-Nyquist (FTN) signaling implemented in the time domain was reported, which offers improved spectral efficiency by introducing a controlled level of inter-symbol interference (ISI) using pulse shaping filters. Moreover, the improvement in energy efficiency was also reported. Note, a frequency domain implementation of FTN is also possible as published in [12]. 
As an alternative multi-carrier modulation format to the aforementioned techniques, $m$-CAP has recently been investigated extensively in VLC systems offering higher data rates and improved spectral efficiency [5], [13]. The working principle of $m$-CAP is based on the splitting the signal bandwidth into equally spaced sub-bands (or subcarriers), thus reducing the effect of low-pass frequency response of an LED on individual subcarriers [14]. In this context, several new methods to optimize the system design and enhance its performance have been successfully reported in the literature. The $m$-CAP system where the subcarriers were spaced unequally within the signal bandwidth was introduced in [15], [16], resulting in improved achievable data rates (up to $36 \%$ ) and reduced computational complexity ( $80 \%$ in terms of real-valued multiplications) at the cost of marginally increased power requirement. In [17], the sub-band index CAP (SI-CAP) was proposed with both spectral and energy efficiency gains over conventional $m$-CAP. Akin to SEFDM, reducing the subcarrier spacing (and hence, the signal bandwidth), purposely overlapping the subcarriers and violating the orthogonality in the frequency domain was successfully implemented in super-Nyquist $m$ CAP ( $m$-SCAP) as was reported by two separate groups in [18]-[20]. Without introducing any additional computational complexity at the receiver, the spectral efficiency gains of $40 \%$ and $25 \%$ for 4 - and 16-level quadrature amplitude modulation (QAM) $m$-SCAP systems, respectively, were experimentally demonstrated in [19]. In [20], a 2-SCAP 16-QAM system with a joint multiple-input multiple-output (MIMO) equalizer at the receiver compensating 5\% overlap (i.e., $11 \%$ gain in spectral efficiency) between adjacent sub-bands was proposed and experimentally investigated.

In [18], [19], we reported the significant bandwidth savings in $m$-SCAP VLC. In this paper, we propose the expanded $m$ SCAP ( $m$-ESCAP) scheme taking advantages of both $m$-CAP and $m$-SCAP. Here, the redundant bandwidth saved thanks to the compression of subcarriers is utilized for signal transmission thus increasing the data throughput. By increasing the baud rate of individual subcarriers, the total signal baud rate is improved while the signal bandwidth requirement remains the same as in conventional $m$-CAP. However, the orthogonality between the subcarriers is still infringed to enhance the system bit rate and the spectral efficiency, which is equivalent to $\mathrm{m}$ SCAP.

Organic polymer LEDs (PLEDs) are emerging as a complementary alternative to conventional LEDs in VLC due to their advantages such as ultra-low-cost manufacturing using wet processing methods, flexible substrates and ability to be patterned into arbitrary photoactive areas [21], [22]. Organic LEDs (OLEDs) are increasingly found in high-end display technologies such as televisions and smart devices, which can be used for the uplink by intensity modulation of the individual pixels of the screen. The main challenge facing the widespread use of this technology in VLC is the relatively low modulation bandwidth of PLED devices as compared to inorganic LEDs. This is mainly due to the low charge transport characteristics of the organic layers, which are significantly lower than for inorganic semiconductors. To date, transmission data rates exceeding $50 \mathrm{Mb} / \mathrm{s}$ have been demonstrated for OFDM and on- off keying VLC links using OLEDs and complex equalization schemes [23], [24].

In this work, we experimentally investigate the performance of the proposed $m$-ESCAP system for a highly bandlimited VLC channel in terms of the transmission data rate, spectral efficiency and bit error rate (BER). Unlike other published works on conventional 1-CAP [4], [25] and $m$-CAP [5], [13], [26]-[28], we compare the system performance with the conventional $m$-CAP [13] and super-Nyquist $m$-SCAP [19] using the same experimental test-bed to clearly emphasize the benefits and drawback of the new system. [19], using the same physical setup to clearly emphasize the benefits and drawbacks of the new system. We report that the $m$-ESCAP scheme supports both higher data rate and spectral efficiency compared to the conventional $m$-CAP at the cost of increased BER. Compared to the $m$-SCAP system, the improvement in measured data rates is also shown, however, at the cost of slightly decreased spectral efficiency and increased BER. Moreover, the newly proposed scheme does not introduce any additional computational complexity and hence can be adopted in bandlimited VLC systems including organic and inorganic devices.

A full system engineering approach, from materials to communication systems, was followed in this work to design the organic VLC link - the design and generation of novel semiconductors and materials [29]-[31], using such materials in the construction of organic optoelectronic devices [32], [33], optoelectronic circuit design [34], [35], communication systems design and signal processing [7]-[9], [13], [19], [27].

The paper is organized as follows. The PLED fabrication process and its electrical characteristics together with the experimental test-bed are described in Section II. The measured results and discussions are given in Section III. Finally, conclusions are drawn in Section IV.

\section{System Under Test}

\section{A. PLED Fabrication Process and Characterization}

The polymer-based device used in this work was fabricated in a nitrogen-filled glovebox by sandwiching a 100 $\mathrm{nm}$ active polymer layer between a transparent prepatterned oxygen plasma-treated indium-tin oxide anode (OSSILA Ltd) [29] covered with a $40 \mathrm{~nm}$ layer of hole-injecting poly $(3,4-$ ethylenedioxythiophene)-poly(styrenesulfonate) (PEDOT:PSS, Heraeus Clevios ${ }^{\mathrm{TM}}$ AI 4083), and a thermally evaporated calcium/aluminum $(30 / 200 \mathrm{~nm})$ metal cathode. We deposited both the PEDOT:PSS interlayer and the active polymer (PIDT 2TPD), whose chemical structure and synthesis we reported recently [30], [31], via spin-coating from solution, following the same procedure used in [30], [36]. The PLEDs consisted of six independent pixels, each with a photoactive area of 4.5 $\mathrm{mm}^{2}$. To prevent the oxidation of the emissive polymer and calcium layer, and therefore to enable us to operate the PLEDs in air, we encapsulated them by covering the metal contacts and the active area with a layer of epoxy resin (OSSILA Ltd) and a protective glass slide (OSSILA Ltd). The glue was then cured by exposing the PLEDs under UV light for 15 minutes in the glovebox. 

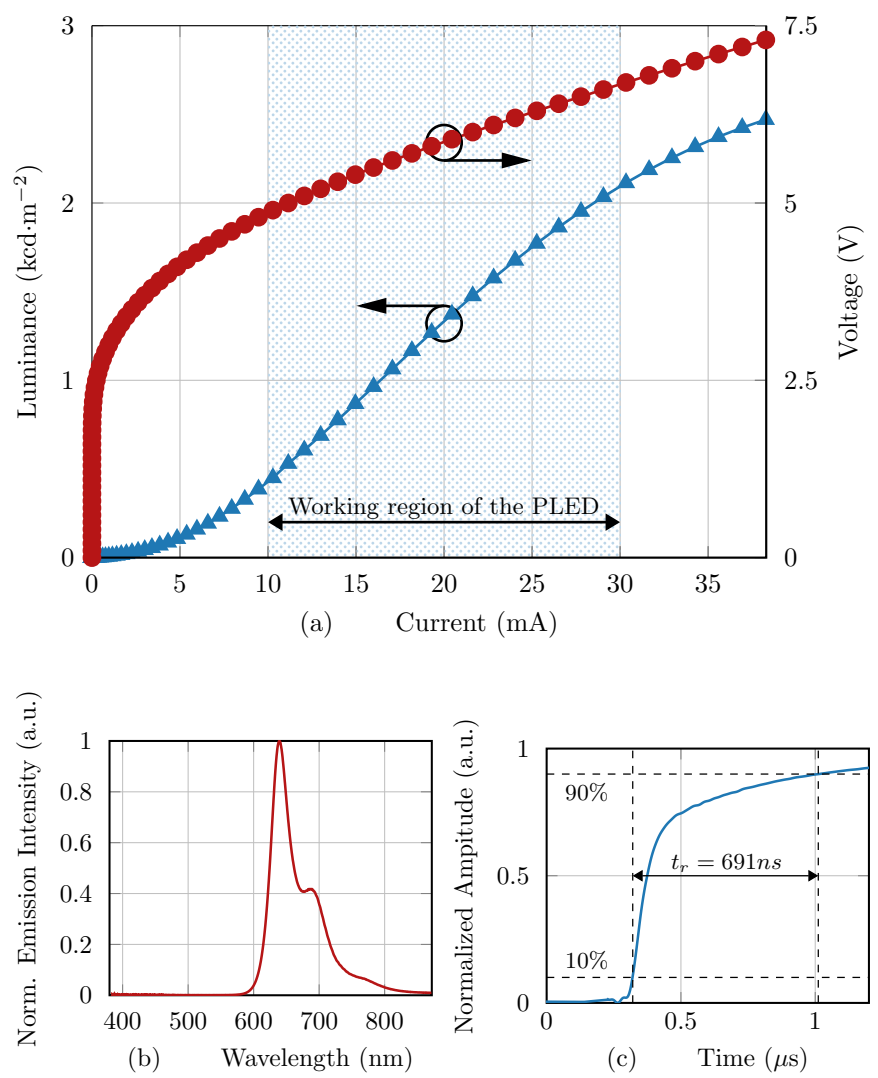

Fig. 1. Electrical characteristics of the PLED: (a) the L-I-V curve, (b) the emission spectrum (peak at $\sim 640 \mathrm{~nm}$ ) and (c) the rise time.

The luminance-current-voltage $(L-I-V)$ curve of the PLED is illustrated in Fig. 1(a) Considering the dc bias current $I_{b}$ of 20 $\mathrm{mA}$ and $4 \mathrm{~V}$ peak-to-peak signal swing (see details in the next section), the PLED was driven in its pseudo-linear operating region within the current range of $\sim 10-30 \mathrm{~mA}$, thus ensuring optimal performance of the system under test. The PLED normalized emission spectrum with the electroluminescence peak at $\sim 640 \mathrm{~nm}$ and the measured rise time $t_{r}$ are shown in Figs. 1(b) and (c), respectively. From Fig. 1(c) $t_{r}=691$ ns and the $3-\mathrm{dB}$ modulation bandwidth of the PLED is $\sim 500$ $\mathrm{kHz}$.

\section{B. Experimental Setup}

1) Transmitter: The schematic block diagram of the proposed system is illustrated in Fig. 2. An independent pseudorandom binary sequence (PRBS) $\boldsymbol{D}_{m}$ of length $10^{5}$ was generated for each subcarrier and mapped onto the $M$-QAM constellation alphabet with the modulation order $M=4$. The signal was up-sampled ('UP' in Fig. 2) by means of zero-padding based on the number of samples-per-symbol $N_{s}=O_{s}\lceil 2 m(1+\beta)\rceil$, where the oversampling factor $O_{s}=4$ without any loss of generality, $\lceil\cdot\rceil$ is the ceiling function and $\beta$ is the excess bandwidth parameter of the pulse shaping filters at the transmitter and receiver (see Fig. 2). After splitting the signal into its real (in-phase) and imaginary (quadrature) components, pulse shaping filters are applied. The filters impulse responses are given as the product of square-root

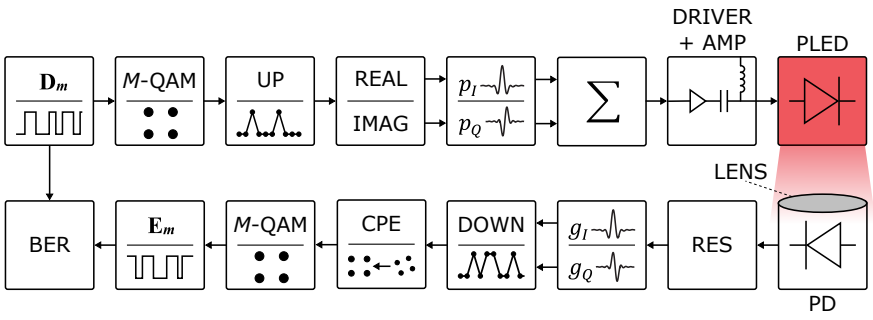

Fig. 2. The experimental setup of the proposed $m$-ESCAP system.

raised cosine (SRRC) filter with a cosine (in-phase) and a sine (quadrature) wave for individual subcarrier $n$ as given by [18], [19]:

$$
\begin{aligned}
& p_{I}^{n}(t)=\left[\frac{\sin [\gamma(1-\beta)]+4 \beta \frac{t}{T_{s}} \cos (\gamma \delta)}{\gamma\left(1-\left(4 \beta \frac{t}{T_{s}}\right)^{2}\right)}\right] \cos \left(2 \pi f_{c}^{n} t\right) \\
& p_{Q}^{n}(t)=\left[\frac{\sin [\gamma(1-\beta)]+4 \beta \frac{t}{T_{s}} \cos (\gamma \delta)}{\gamma\left(1-\left(4 \beta \frac{t}{T_{s}}\right)^{2}\right)}\right] \sin \left(2 \pi f_{c}^{n} t\right)
\end{aligned}
$$

where $T_{s}$ is the symbol duration, $\gamma=\pi t / T_{s}$ and $\delta=1+\beta$. Clearly, the carrier frequencies $f_{c}^{n}$ of individual subcarriers are determined by the impulse responses of pulse shaping transmit filters as:

$$
f_{c}^{n}=B_{s}(1+\alpha)\left(\frac{1}{2 m}-\frac{(n-1)(1+\alpha m-m)}{m(m-1)}\right)
$$

where $B_{s}$ is the bandwidth of transmitted signal, recalling $m$ is the total number of sub-bands and the subcarrier index is given by $n=1,2, \ldots, m$. Note, $B_{s}$ for $m$-ESCAP and conventional $m$-CAP are the same whereas in $m$-SCAP the utilized $B_{s}$ is reduced by $\alpha$. To improve the data rate while maintaining the same $B_{s}$, the individual sub-bands must be expanded, which is achieved by increasing baud rates of the subcarriers, thus resulting in overlapping between them. The subcarrier baud rate for $m$-ESCAP is given as:

$$
R_{s, \text { sub }}=\frac{B_{s}(1+\alpha)}{m(1+\beta)}
$$

The concept of the proposed scheme is best illustrated using the frequency spectrum as shown in Fig. 3 for $m=2$ and $\alpha=20 \%$. As can be seen, the bandwidth occupied by $m$ SCAP (dashed line) is reduced by $\alpha$ in contrast to $m$-ESCAP. On the other hand, the increased $R_{s, s u b}$ in $m$-ESCAP leads to the higher bandwidth requirement (equal to that of conventional $m$-CAP) while still breaking the orthogonality between subcarriers. Note, the factor $\alpha$ is used interchangeably to indicate signal modification, either for bandwidth compression ( $m$-SCAP), or for the bandwidth expansion ( $m$-ESCAP) when discussing either format.

Finally, the generated signal is expressed by:

$$
s(t)=\sum_{n=1}^{m}\left(s_{I}^{n}(t) * p_{I}^{n}(t)-s_{Q}^{n}(t) * p_{Q}^{n}(t)\right)
$$




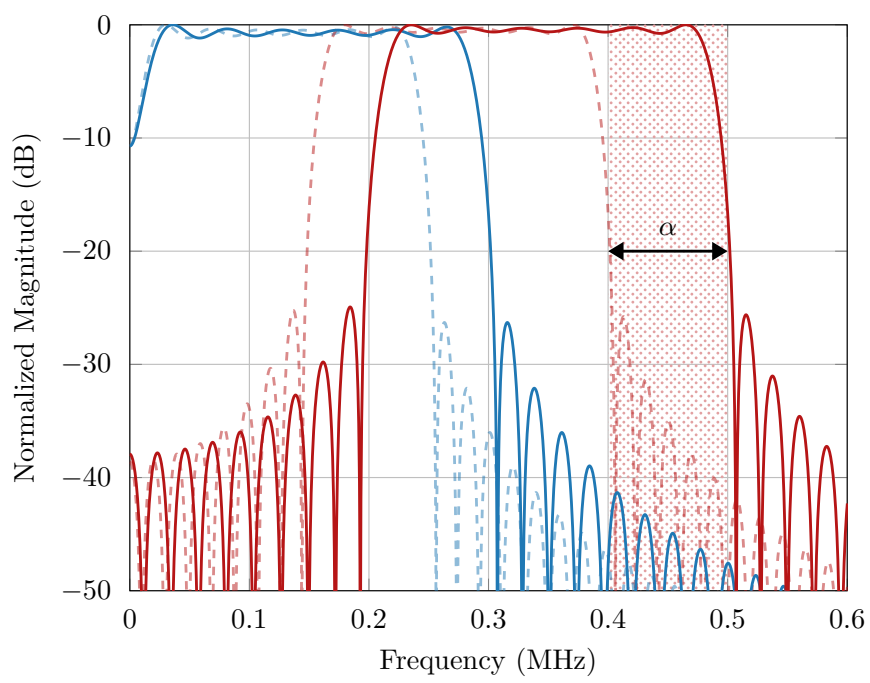

Fig. 3. The ideal spectra of the transmit filters of 2-ESCAP system with $\alpha=20 \%$ (solid line) showing the full bandwidth allocation and overlapping between the sub-bands (depicted in blue and red). For a comparison, $m$-SCAP system is also shown (dashed line).

where $s_{I}^{n}(t)$ and $s_{Q}^{n}(t)$ are in phase and quadrature components of the $M$-QAM signal for the nth subcarrier, respectively and $*$ denotes the time domain convolution. The output signal with the sampling frequency $f_{s}=R_{s, s u b} N_{s}$ was loaded into a Tektronix AFG90002A arbitrary waveform generator (AWG) the output of which was amplified by 4 times to $2 \mathrm{~V}$ ac using a Texas Instruments THS3202 current-feedback amplifier and then dc level shifted by $20 \mathrm{~mA}$ prior to intensity modulation of a red PLED (see Fig. 1(a). The bandwidth of the link was set to $500 \mathrm{kHz}$ (equal to the modulation bandwidth of the PLED, see $t_{r}=691 \mathrm{~ns}$ in Fig. 1(c) to ensure no high-frequency attenuation of the subcarriers and the dominant noise source tested is the ICI between sub-bands.

2) Receiver: At the receiver, a fixed gain Thorlabs PDA10A2 optical photodetector (with mounted focusing aspheric lens with a focal length $4.51 \mathrm{~mm}$ ) with a transimpedance amplifier was placed at a $5 \mathrm{~cm}$ distance from the transmitter. Note, the short transmission range is due to the limited output power of the PLED. The output of the optical receiver was captured using a real time oscilloscope Tektronix MSO80604B at a sampling frequency of $62.5 \mathrm{MHz}$ and saved for data processing in MATLAB. The signal was resampled to match the original sampling frequency ('RES') and passed through the time-reversed receiver filters following $g_{I}^{n}(t)=p_{I}^{n}(-t)$ for the in-phase and $g_{Q}^{n}(t)=p_{Q}^{n}(-t)$ for the quadrature components of the signal, respectively. Next, the signal was down-sampled ('DOWN') and a common phase error (CPE) correction algorithm was applied on the received constellations. Finally, the $M$-QAM symbols were recovered and BER of the VLC link was determined by comparing the transmitted and regenerated (i.e., estimated) bits $\boldsymbol{E}_{\boldsymbol{m}}$.

Note, the proposed system under test is designed with the signal bandwidth equal to the PLED bandwidth. Unlike OFDM using a cyclic prefix $(\mathrm{CP})$ to combat ISI, in $m$-CAP ISI is limited and controlled by the excess energy factor of the pulse shaping and matched filters. In addition, no equalizers are utilized to investigate the fundamental performance of the proposed scheme. The main interference is the ICI (generated by the non-orthogonal subcarriers), which can be minimized by utilizing a hybrid iterative and sphere decoder at the cost of increased receiver complexity as in [7].

\section{RESUlTS AND DiscUSSION}

In this section, the measured results are given and discussed, recalling the parameters used in experiments were: $m=10$, $M=4, \beta=\{0.1,0.5\}, \alpha=\{10,20,30,40\} \%$ and $B_{s}=0.5$ $\mathrm{MHz}$. Note that, the parameters adopted here are based on the results reported in our previous works: $\alpha$ and $\beta$ values are selected based on our previous studies reported in [18], [19], [27], while for $m>10$ only marginal performance improvement is achieved as shown in [5], [14]. All the systems including conventional $m$-CAP, $m$-SCAP and $m$-ESCAP are compared in terms of BER, bit rate $R_{b}$ and spectral efficiency $\eta_{s e}$.

The measured BER as a function of the compression/expansion level $\alpha$ for the systems under test is illustrated in Figs. 4(a) and (b) for $\beta=0.1$ and 0.5, respectively. For $\beta=0.1, m$-SCAP outperforms $m$-ESCAP in terms of the BER by approximately one order of magnitude for $\alpha=10 \%$, while for higher values of $\alpha$, both schemes show similar results. This is because the $m$-ESCAP signal has slightly higher bandwidth requirement and hence experiences additional attenuation compared to $m$-SCAP system. Note, for $\alpha \geq 20 \%$ the ICI is dominant compared to the noise and similar BERs are reported as in [19]. Note the sharp increase in the BER for $\alpha>10 \%$ is mainly due to the lower value of $\beta$ (i.e., reduced excess bandwidth and steeper frequency response of individual pulse shaping filters). Both systems support data transmission with a BER below the $7 \%$ forward error correction (FEC) limit of $3.8 \cdot 10^{-3}$ for $\alpha=10 \%$, where $R_{b}$ and $\eta_{s e}$ are $1 \mathrm{Mb} / \mathrm{s}$ and $2 \mathrm{~b} / \mathrm{s} / \mathrm{Hz}$, respectively, for $m$-ESCAP. For the same $\alpha$, $\eta_{s e}$ is $2.02 \mathrm{~b} / \mathrm{s} / \mathrm{Hz}$ for $m$-SCAP, however the overall $R_{b}$ is reduced by about $10 \%$ to $0.91 \mathrm{Mb} / \mathrm{s}$ with the advantage of marginally improved BER performance. The insets display the constellation diagrams of the $1^{\text {st }}$ subcarrier for both systems in their respective colors for $\alpha=10 \%$.

For $\beta=0.5$, the BER performance of both systems is depicted in Fig. 4(b). The $m$-SCAP scheme supports transmission with BERs below the 7\% FEC limit for $\alpha$ up to $30 \%$ (i.e., error free for $\alpha=10 \%$ and $20 \%$ ) with the measured $R_{b}$ of 0.67 $\mathrm{Mb} / \mathrm{s}$ and $\eta_{s e}$ of $1.9 \mathrm{~b} / \mathrm{s} / \mathrm{Hz}$. In contrast, $m$-ESCAP can support BERs below 7\% FEC for $\alpha \leq 20 \%$ offering $R_{b}$ and $\eta_{s e}$ of 0.8 $\mathrm{Mb} / \mathrm{s}$ and $1.6 \mathrm{~b} / \mathrm{s} / \mathrm{Hz}$, respectively. Note that, the gain in $R_{b}$ of $\sim 19 \%$ is achieved at the cost of reduced $\eta_{s e}$ (i.e., by about $16 \%)$ and BER performance. In both schemes, the achievable value of $\alpha$ is enhanced in comparison to the previous case (i.e., $\beta=0.1$ ) since a higher excess bandwidth is utilized, acting as a guard band and therefore reducing the effect of the ICI on the transmitted signal. Thus, a larger overlapping between subcarriers can be supported. Considering $\alpha=30 \%$, the BER of $m$-ESCAP is decreased however still remains below the $20 \%$ FEC limit, thus supporting $R_{b}$ of $0.87 \mathrm{Mb} / \mathrm{s}$ ( $\sim 30 \%$ improvement in comparison to $m$-SCAP) and $\eta_{s e}$ of 

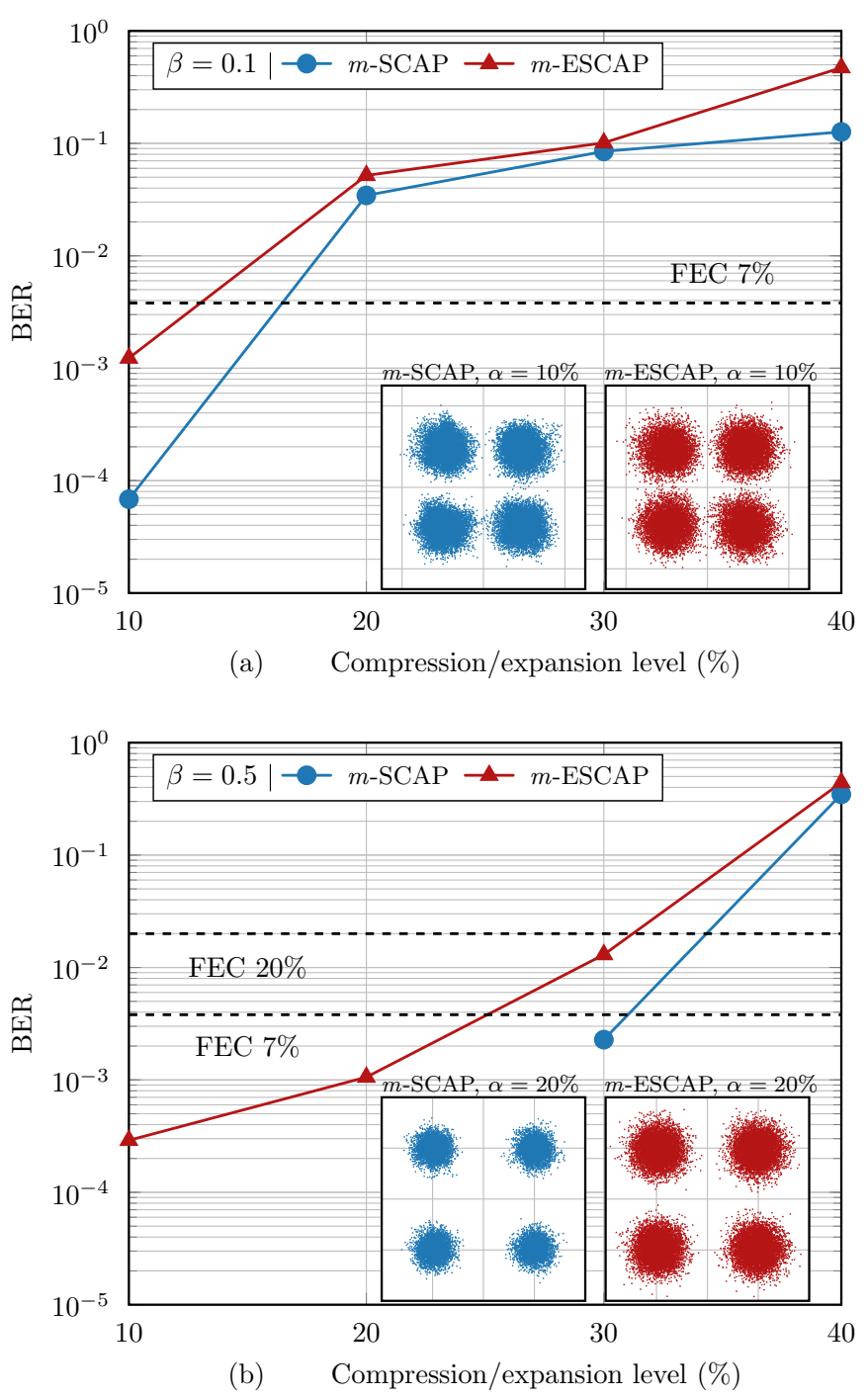

Fig. 4. The measured BER perofrmance of $m$-SCAP and $m$-ESCAP systems for a range of $\alpha$ and for: (a) $\beta=0.1$, and (b) $\beta=0.5$. Inset are the received constellation diagrams of the $1^{\text {st }}$ subcarriers for both systems in their respective colors for: (a) $\alpha=10 \%$ and (b) $\alpha=20 \%$.

$1.73 \mathrm{~b} / \mathrm{s} / \mathrm{Hz}$ ( $\sim 9 \%$ deterioration). The received constellation diagrams of the $1^{\text {st }}$ subcarriers in both systems under test for $\alpha=20 \%$ are shown in the inset in Fig. 4(b).

Next, we compare the proposed scheme with the conventional orthogonal $m$-CAP system with no compression (i.e., $\alpha=0 \%$ ). For $\beta=0.1$ and $\alpha=10 \%$, the measured improvement of $\sim 10 \%$ in both $R_{b}$ and $\eta_{s e}$ for $m$-ESCAP scheme is reported compared to conventional $m$-CAP $\left(R_{b}=0.91 \mathrm{Mb} / \mathrm{s}\right.$ and $\eta_{s e}=1.82 \mathrm{~b} / \mathrm{s} / \mathrm{Hz}$ ). With higher excess bandwidth (i.e., $\beta=0.5)$ and $\alpha=20 \%, R_{b}$ and $\eta_{s e}$ are enhanced by about $\sim 10 \%$ and $20 \%$, respectively, in comparison to $R_{b}=0.67$ $\mathrm{Mb} / \mathrm{s}$ and $\eta_{s e}=1.33 \mathrm{~b} / \mathrm{s} / \mathrm{Hz}$ supported by the orthogonal scheme. In each case, the measured improvement comes at the cost of higher BER as expected.

The summary of the measured $R_{b}$ (solid lines) and $\eta_{s e}$ (dashed lines) of all the systems under test against $\alpha$ for $\beta=0.1$ and 0.5 is depicted in Figs. 5(a) and (b) respectively. Also shown are the 7\% FEC BER limits (black dashed line).
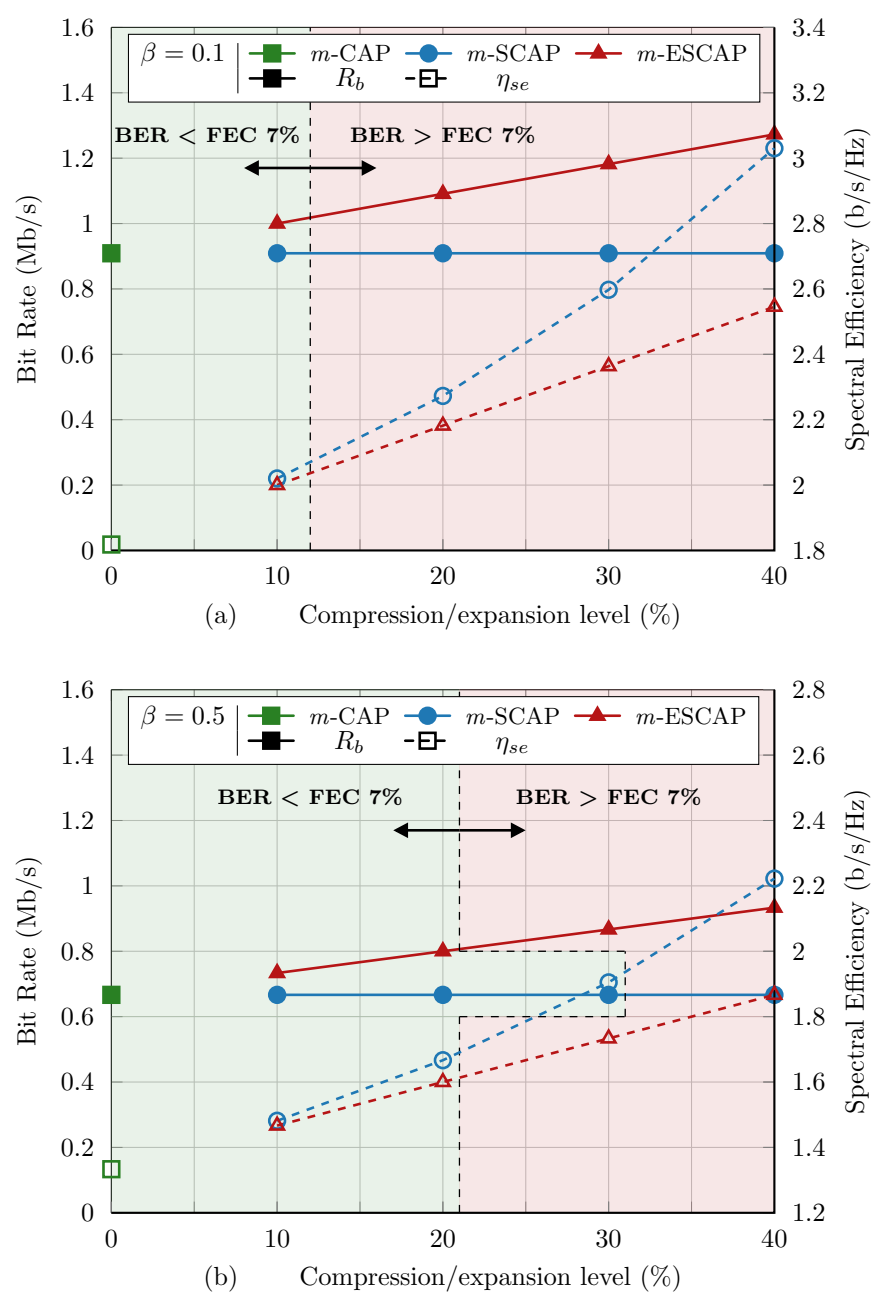

Fig. 5. Measured bit rates (solid lines) and spectral efficiencies (dashed lines) of the tested systems with highlighted 7\% FEC BER target for a range of $\alpha$ for: (a) $\beta=0.1$ and (b) $\beta=0.5$.

Next, we investigate the signal-to-noise and interference ratio (SNIR) performance of the proposed schemes against individual subcarriers as depicted in Figs. 6(a) and (b) for $\beta=0.1, \alpha=10 \%$ and $\beta=0.5$ and $\alpha=20 \%$ and $30 \%$, respectively. The SNIR is determined from the measured root mean square error vector magnitude (EVM) from the captured constellation diagrams as given by [37]:

$$
S N I R=\frac{1}{E V M_{r m s}^{2}}
$$

For $\beta=0.1$, the measured SNIR of both $m$-SCAP and $m$-ESCAP systems for $\alpha=10 \%$ is significantly decreased compared to the conventional $m$-CAP as expected due to the ICI, particularly in the center sub-bands, which experience interference from either side. For instance, at $n=5$, the SNIR values are dropped by $\sim 6 \mathrm{~dB}$ and $4.5 \mathrm{~dB}$ for $m$-ESCAP and $m$-SCAP, respectively, compared to $m$-CAP, see Fig. 6(a) Moreover, $m$-ESCAP shows the lowest SNIR requirement (i.e., an average of $10 \mathrm{~dB}$ ) with a $1 \mathrm{~dB}$ power penalty for subbands $n=1-6$ which increases to $>3 \mathrm{~dB}$ for $n=7-10$. The SNIR degrades with increasing $n$ due to the higher bandwidth requirement of the proposed system in comparison to $\mathrm{m}$ - 

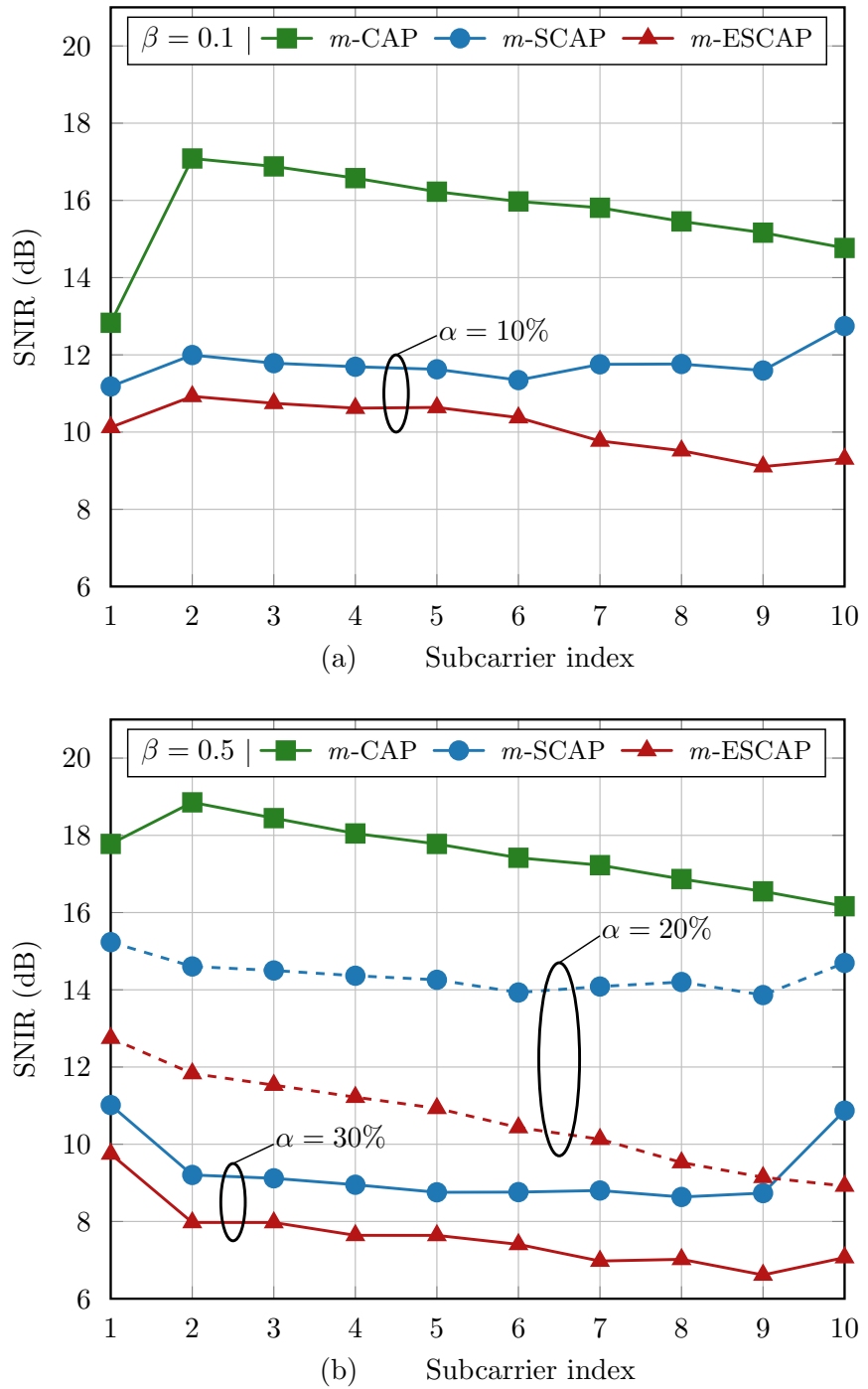

Fig. 6. The measured SNIR performance for individual subcarriers for (a) $\beta=0.1, \alpha=10 \%$ and (b) $\beta=0.5, \alpha=20 \%$ and $30 \%$. The conventional $m$-CAP system (green solid line) is shown as a reference.

SCAP. A similar trend is observed in Fig. 6(b) for $\beta=0.5$ and $\alpha=20 \%$ and $30 \%$. The measured values correspond to the BER performance illustrated in Fig. 4(b) For higher compression levels (i.e., $\alpha=30 \%$ ), the SNIR is severely reduced and ICI becomes the dominant term causing the link performance degradation. Note, for $\alpha=20 \%$ and $30 \%$ the measured SNIR values are marginally improved for $n=1$ and 10 since they interfere with only a single adjacent subcarrier.

\section{CONCLUSION}

In this paper, we proposed and experimentally verified the $m$-ESCAP modulation scheme for a highly bandlimited organic VLC system, which utilizes non-orthogonal subcarriers for signal transmission having the bandwidth requirement equal to that of traditional $m$-CAP. Due to the low PLED bandwidth, improving spectral efficiency is an important step toward achieving high data rate links. We demonstrated gains in the measured bit rate and spectral efficiency of $\sim 10 \%$ in comparison to $m$-CAP. The bit rate was also improved (by nearly 20\%) when compared to $m$-SCAP scheme, however loss in the spectral efficiency needs to be expected. For all the gains reported BER was kept below 7\% FEC limit. The comparison of several $m$-CAP based VLC systems is the focus of future work while optimizing the systems performance using bit- and power-loading algorithms.

\section{REFERENCES}

[1] X. Huang, S. Chen, Z. Wang, J. Shi, Y. Wang, J. Xiao, and N. Chi, "2.0$\mathrm{Gb} / \mathrm{s}$ Visible Light Link Based on Adaptive Bit Allocation OFDM of a Single Phosphorescent White LED," IEEE Photonics Journal, vol. 7, no. 5, pp. 1-8, 2015.

[2] Y. Wang, L. Tao, X. Huang, J. Shi, and N. Chi, "8-Gb/s RGBY LEDBased WDM VLC System Employing High-Order CAP Modulation and Hybrid Post Equalizer," IEEE Photonics Journal, vol. 7, no. 6, pp. 1-7, 2015.

[3] F. M. Wu, C. T. Lin, C. C. Wei, C. W. Chen, Z. Y. Chen, H. T. Huang, and S. Chi, "Performance Comparison of OFDM Signal and CAP Signal Over High Capacity RGB-LED-Based WDM Visible Light Communication," IEEE Photonics Journal, vol. 5, no. 4, p. 7901507, 2013.

[4] N. Chi, Y. Zhou, S. Liang, F. Wang, J. Li, and Y. Wang, "Enabling Technologies for High-Speed Visible Light Communication Employing CAP Modulation," Journal of Lightwave Technology, vol. 36, no. 2, pp. 510-518, 2018.

[5] K. Werfli, P. Chvojka, Z. Ghassemlooy, N. Hassan, S. Zvanovec, A. Burton, P. Haigh, and M. Bhatnagar, "Experimental Demonstration of High-Speed $4 \times 4$ Imaging Multi-CAP MIMO Visible Light Communications," Journal of Lightwave Technology, vol. 36, no. 10, 2018.

[6] M. Rodriguez and I. Darwazeh, "A spectrally efficient frequency division multiplexing based communications system," in 8th International OFDM Workshop, Hamburg, 2003, pp. 70-74.

[7] I. Darwazeh, T. Xu, T. Gui, Y. Bao, and Z. Li, "Optical SEFDM System; Bandwidth Saving Using Non-Orthogonal Sub-Carriers," IEEE Photonics Technology Letters, vol. 26, no. 4, pp. 352-355, 2014.

[8] M. Rodriguez and I. Darwazeh, "Fast OFDM: A proposal for doubling the data rate of OFDM schemes," in IEEE/IEE International Conference on Telecommunications (ICT 2002), 2002, pp. 484-487.

[9] P. A. Haigh and I. Darwazeh, "Visible light communications: Fastorthogonal frequency division multiplexing in highly bandlimited conditions," in 2017 IEEE/CIC International Conference on Communications in China (ICCC Workshops), Qingdao, 2017, pp. 1-8.

[10] J. Fan, S. Guo, X. Zhou, Y. Ren, G. Y. Li, and X. Chen, "Faster-ThanNyquist Signaling: An Overview," IEEE Access, vol. 5, pp. 1925-1940, 2017.

[11] J. B. Anderson, F. Rusek, and V. Öwall, "Faster-Than-Nyquist Signaling," Proceedings of the IEEE, vol. 101, no. 8, pp. 1817-1830, 2013.

[12] F. Rusek and J. B. Anderson, "The two dimensional Mazo limit," in Proceedings. International Symposium on Information Theory, 2005. ISIT 2005., 2005, pp. 970-974.

[13] P. Haigh, A. Burton, K. Werfli, H. Minh, E. Bentley, P. Chvojka, W. Popoola, I. Papakonstantinou, and S. Zvanovec, "A Multi-CAP Visible-Light Communications System with $4.85-\mathrm{b} / \mathrm{s} / \mathrm{Hz}$ Spectral Efficiency," IEEE Journal on Selected Areas in Communications, vol. 33, no. 9, pp. 1771-1779, 2015.

[14] P. Haigh, S. Le, S. Zvanovec, Z. Ghassemlooy, P. Luo, T. Xu, P. Chvojka, T. Kanesan, E. Giacoumidis, P. Canyelles-Pericas, H. Minh, W. Popoola, S. Rajbhandari, I. Papakonstantinou, and I. Darwazeh, "Multi-band carrier-less amplitude and phase modulation for bandlimited visible light communications systems," IEEE Wireless Communications, vol. 22, no. 2, pp. 46-53, 2015.

[15] P. Chvojka, S. Zvanovec, K. Werfli, P. Haigh, and Z. Ghassemlooy, "Variable $m$-CAP for bandlimited Visible Light Communications," in 2017 IEEE International Conference on Communications Workshops, ICC Workshops 2017, Paris, 2017, pp. 1-5.

[16] K. Werfli, P. A. Haigh, Z. Ghassemlooy, N. B. Hassan, and S. Zvanovec, "A new concept of multi-band carrier-less amplitude and phase modulation for bandlimited visible light communications," in 2016 10th International Symposium on Communication Systems, Networks and Digital Signal Processing (CSNDSP), Prague, 2016, pp. 1-5.

[17] K. O. Akande and W. O. Popoola, "Subband Index Carrierless Amplitude and Phase Modulation for Optical Communications," Journal of Lightwave Technology, vol. 36, no. 18, pp. 4190-4197, 2018. 
[18] P. Haigh, P. Chvojka, Z. Ghassemlooy, S. Zvanovec, and I. Darwazeh, "Non-Orthogonal Multi-band CAP for Highly Spectrally Efficient VLC Systems," in 2018 11th International Symposium on Communication Systems, Networks and Digital Signal Processing, CSNDSP 2018, 2018.

[19] P. A. Haigh, P. Chvojka, Z. Ghassemlooy, S. Zvanovec, and I. Darwazeh, "Visible Light Communications: Multi-band Super-Nyquist CAP Modulation," Optics Express, vol. 27, no. 6, pp. 8912-8919, 2019.

[20] S. Liang, L. Qiao, X. Lu, and N. Chi, "Enhanced performance of a multiband super-Nyquist CAP16 VLC system employing a joint MIMO equalizer," Optics Express, vol. 26, no. 12, pp. 15 718-15 725, 2018.

[21] B.-J. de Gans, P. C. Duineveld, and U. S. Schubert, "Inkjet Printing of Polymers: State of the Art and Future Developments," Advanced Materials, vol. 16, no. 3, pp. 203-213, 2004.

[22] W. Gaynor, S. Hofmann, M. G. Christoforo, C. Sachse, S. Mehra, A. Salleo, M. D. McGehee, M. C. Gather, B. Lüssem, L. MüllerMeskamp, P. Peumans, and K. Leo, "Color in the Corners: ITOFree White OLEDs with Angular Color Stability," Advanced Materials, vol. 25, no. 29, pp. 4006-4013, 2013.

[23] H. Chen, Z. Xu, Q. Gao, and S. Li, "A 51.6 Mb/s Experimental VLC System Using a Monochromic Organic LED," IEEE Photonics Journal, vol. 10, no. 2, pp. 1-12, 2018.

[24] P. A. Haigh, F. Bausi, H. Le Minh, I. Papakonstantinou, W. O. Popoola, A. Burton, and F. Cacialli, "Wavelength-Multiplexed Polymer LEDs: Towards $55 \mathrm{Mb} / \mathrm{s}$ Organic Visible Light Communications," IEEE Journal on Selected Areas in Communications, vol. 33, no. 9, pp. 1819-1828, 2015.

[25] K. O. Akande, P. A. Haigh, and W. O. Popoola, "On the Implementation of Carrierless Amplitude and Phase Modulation in Visible Light Communication," IEEE Access, vol. 6, pp. 60 532-60 546, 2018.

[26] P. A. Haigh, P. Chvojka, S. Zvanovec, Z. Ghassemlooy, and I. Darwazeh, "Analysis of Nyquist Pulse Shapes for Carrierless Amplitude and Phase Modulation in Visible Light Communications," Journal of Lightwave Technology, vol. 36, no. 20, pp. 5023-5029, 2018.

[27] P. Chvojka, K. Werfli, S. Zvanovec, P. Haigh, V. Vacek, P. Dvorak, P. Pesek, and Z. Ghassemlooy, "On the $m$-CAP Performance with Different Pulse Shaping Filters Parameters for Visible Light Communications," IEEE Photonics Journal, vol. 9, no. 5, pp. 1-12, 2017.

[28] W. Niu, Y. Ha, and N. Chi, "Novel Phase Estimation Scheme Based On Support Vector Machine for Multiband-CAP Visible Light Communication System," in 2018 Asia Communications and Photonics Conference (ACP), 2018, pp. 1-3.

[29] T. M. Brown, G. M. Lazzerini, L. J. Parrott, V. Bodrozic, L. Bürgi, and F. Cacialli, "Time dependence and freezing-in of the electrode oxygen plasma-induced work function enhancement in polymer semiconductor heterostructures," Organic Electronics, vol. 12, no. 4, pp. 623-633, 2011.
[30] A. Minotto, P. Murto, Z. Genene, A. Zampetti, G. Carnicella, W. Mammo, M. R. Andersson, E. Wang, and F. Cacialli, "Efficient Near-Infrared Electroluminescence at $840 \mathrm{~nm}$ with Metal-Free SmallMolecule:Polymer Blends," Advanced Materials, vol. 30, no. 34, p. 1706584, 2018

[31] P. Murto, Z. Genene, C. M. Benavides, X. Xu, A. Sharma, X. Pan, O. Schmidt, C. J. Brabec, M. Andersson, W. Mammo, S. F. Tedde, and E. Wang, "High Performance All-Polymer Photodetector Comprising a DonorAcceptorAcceptor Structured IndacenodithiopheneBithieno[3,4c]Pyrroletetrone Copolymer," ACS Macro Letters, vol. 7, no. 4, pp. 395400, 2018.

[32] A. Zampetti, A. Minotto, and F. Cacialli, "Near-Infrared (NIR) Organic Light-Emitting Diodes (OLEDs): Challenges and Opportunities," Advanced Functional Materials, vol. 29, no. 21, p. 1807623, 2019.

[33] A. Zampetti, A. Minotto, B. M. Squeo, V. G. Gregoriou, S. Allard, U. Scherf, C. L. Chochos, and F. Cacialli, "Highly Efficient Solid-State Near-infrared Organic Light-Emitting Diodes incorporating A-D-A Dyes based on $\alpha, \beta$-unsubstituted "BODIPY" Moieties," Scientific Reports, vol. 7, no. 1, p. 1611, 2017.

[34] P. A. Haigh, Z. Ghassemlooy, S. Rajbhandari, and I. Papakonstantinou, "Visible light communications using organic light emitting diodes," IEEE Communications Magazine, vol. 51, no. 8, pp. 148-154, 2013.

[35] B. Wilson and I. Darwazeh, "Transimpedance optical preamplifier with a very low input resistance," Electronics Letters, vol. 23, no. 4, pp. $138-139,1987$.

[36] S. T. Le, T. Kanesan, F. Bausi, P. A. Haigh, S. Rajbhandari, Z. Ghassemlooy, I. Papakonstantinou, W. O. Popoola, A. Burton, H. L. Minh, F. Cacialli, and A. D. Ellis, " $10 \mathrm{Mb} / \mathrm{s}$ visible light transmission system using a polymer light-emitting diode with orthogonal frequency division multiplexing," Optics Letters, vol. 39, no. 13, pp. 3876-3879, 2014.

[37] R. A. Shafik, M. S. Rahman, and A. R. Islam, "On the Extended Relationships Among EVM, BER and SNR as Performance Metrics," in 2006 International Conference on Electrical and Computer Engineering, 2006, pp. 408-411. 